\title{
SUPPORT THEOREMS FOR TOTALLY GEODESIC RADON TRANSFORMS ON CONSTANT CURVATURE SPACES
}

\author{
ÁRPÁD KURUSA
}

(Communicated by J. Marshall Ash)

\begin{abstract}
We prove a relation between the $k$-dimensional totally geodesic Radon transforms on the various constant curvature spaces using the geodesic correspondence between the spaces. Then we use this relation to obtain improved support theorems for these transforms.
\end{abstract}

\section{INTRODUCTION}

Let $\mathscr{M}^{n}$ be an $n$-dimensional simply connected Riemannian manifold of constant curvature $\kappa$. Normalizing the metric so that $\kappa=-1,0$, or +1 we must deal only with the hyperbolic space $\mathbb{H}^{n}(\kappa=-1)$, the Euclidean space $\mathbb{R}^{n} \quad(\kappa=0)$, and the sphere $S^{n}(\kappa=+1)$.

For a fixed $k(1 \leq k \leq n-1)$ let $\xi$ be an arbitrary totally geodesic submanifold of $\mathscr{K}^{n}$ of dimension $k$. The $k$-dimensional totally geodesic Radon transform $R f$ of $f \in L^{2}\left(\mathscr{M}^{n}\right)$ is defined by

$$
R f(\xi)=\int_{\xi} f(x) d x,
$$

where $d x$ is the surface element on $\xi$ induced by the metric of $\mathscr{M}^{n}[6]$.

Our first goal in this paper is to prove a link between these Radon transforms taken on different constant curvature spaces. The proof is based on the geodesic correspondence between the spaces [10]. The idea to use projection from constant curvature spaces to Euclidean spaces also appeared in $[3,11]$.

The connection established in Theorem 2.1 allows one to transpose results from one space to the other; hence, it can be used to get inversion formulas, range characterizations [1], and so on. We shall use it to obtain support theorems.

Roughly speaking a support theorem states that if the function $f$ is in a suitable function space of $\mathscr{M}^{n}$ and the support of $R f, \operatorname{supp} R f$, is bounded, then supp $f \subseteq P$ supp $R f$, where $P$ maps the set of total geodesics into $\mathscr{M}^{n}$ so that the total geodesics correspond to their point closest to the origin. For more information about the applications of support theorems we refer to $[4,5$, 7].

Received by the editors April 11, 1991 and, in revised form, January 4, 1993.

1991 Mathematics Subject Classification. Primary 44A05, 53C65.

Key words and phrases. Radon transform, constant curvature spaces. 
We consider the cases $k=n-1$ and $k \leq n-2$ separately, because of their principal differences.

In the case $k=n-1$, Helgason's support theorem for the Euclidean space [5] via Theorem 2.1 implies significant improvement on his support theorem for the hyperbolic space [5] replacing the rapid decrease by a decrease of order $n$. Transposing Helgason's support theorem to the sphere we find the support theorem for $C^{\infty}$ functions vanishing with all their derivatives at the equator.

For the case $k \leq n-2$, we use Schneider's result [12] to obtain a support theorem on the sphere. After transposing this to the other spaces we find the support theorem on all the constant curvature spaces supposing only a finite order decrease on the functions, instead of the rapid decrease.

\section{Preliminaries}

In this section we give the most necessary preliminaries and theorems upon which $\S 2$ is based. Most of the facts listed here are proved in [5, 8, 9, 10, 14].

The Riemannian metric on $\mathscr{M}^{n}$, one of the three spaces of constant curvature, is completely described by the 'size function' $\nu: \mathbb{R}_{+} \rightarrow \mathbb{R}_{+}$. The size function $\nu$ determines the radius $\nu(r)$ of the Euclidean sphere in $\mathbb{R}^{n}$ that is isometric to the geodesic sphere of radius $r$ in $\mathscr{M}^{n}$ [14].

An other important function on the constant curvature spaces is the 'projector function' $\mu: \mathbb{R}_{+} \rightarrow \mathbb{R}_{+}$. This function generates a geodesic correspondence between the constant curvature spaces and the Euclidean space via the geodesic polar coordinatization [10]. Take a fixed point in $\mathscr{M}^{n}$, say $O$, and the origin in $\mathbb{R}^{n}$. Then the map that makes the geodesic correspondence between these spaces is

$$
\tilde{\mu}: \mathscr{M}^{n} \rightarrow \mathbb{R}^{n} \quad\left(\operatorname{Exp}_{O} r \omega \mapsto \mu(r) \omega\right),
$$

where $\omega$ is a unit vector in $T_{O} \mathscr{M}^{n}, r \in \mathbb{R}_{+}$, and $\mathbb{R}^{n}$ is identified with $T_{O} \mathscr{M}^{n}$. Further on we shall use only the open half sphere $\mathbb{P}^{n}$ having the center point $O$ rather than the whole sphere $S^{n}$ because the odd functions obviously have zero Radon transform on the sphere [5].

From Figure 1 one can easily read off the entries of Table 1.

The point is that the geodesics in $\mathscr{M}^{n}$ are precisely the nonempty intersections of $\mathscr{M}^{n}$ with the two planes through the origin of $\mathbb{R}^{n+1}$.

We shall need the following easy consequence of Schneider's result and Helgason's support theorem for the Euclidean space, which we recall after the proof.

Lemma 1.1. Let $k \leq n-2, B$ be a spherical cap, and the function $f \in C\left(S^{n}\right)$ be symmetric. If the integral of $f$ is zero over all the $k$-dimensional great subspheres $\xi$ not intersecting the spherical cap $B$, then $f$ is zero outside $B$ and its antipodal spherical cap.

Proof. Let $S^{k+1}$ be such an intersection of $S^{n}$ with a $(k+2)$-dimensional subspace of $\mathbb{R}^{n+1}$ that does not intersect the spherical cap $B$. Then no $k$ dimensional great subsphere $\xi$ of $S^{k+1}$ intersects $B$; hence, all the integrals of $f \in C\left(S^{n}\right)$ over the hypersubspheres of $S^{k+1}$ vanish. Therefore, $f \equiv 0$ on $S^{k+1}$ by [12], which proves the lemma.

Theorem 1.2 [5, Theorem 2.6]. Let $f \in C\left(\mathbb{R}^{n}\right)$ satisfy the following conditions:

(1) For each integer $m>0,|x|^{m} f(x)$ is bounded on $\mathbb{R}^{n}$.

(2) For each hyperplane $\xi$ outside the unit ball, $|x|<1, R f(\xi)=0$.

Then $f(x)=0$ for $|x|>1$. 


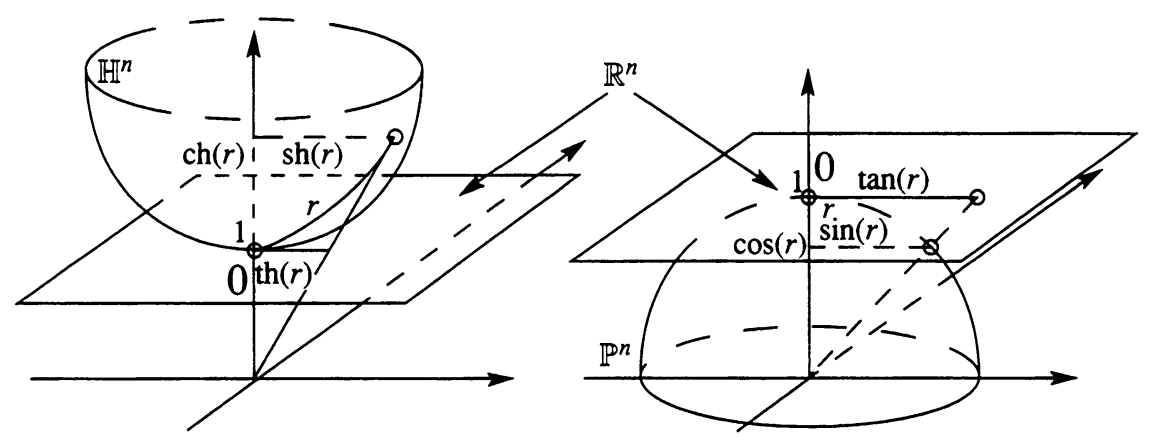

FIGURE 1. The geometric meaning of the function $\tilde{\mu}$ can be read off this figure, where the quadratic model of the hyperbolic space and the sphere in $\mathbb{R}^{n+1}$ [5] is 'projected' to the hyperplane $\mathbb{R}^{n}$, determined by the equation $x_{n+1}=1$, by the straight lines through the origin of $\mathbb{R}^{n+1}$. For further details see the subject of the projective realization of constant curvature spaces in standard textbooks.

TABLE 1

\begin{tabular}{|c||c|c|c|}
\hline $\mathscr{M}^{n}$ & $\kappa$ & $\nu$ & $\mu$ \\
\hline \hline $\mathbb{H}^{n}$ & -1 & $\sinh r$ & $\tanh r$ \\
$\mathbb{R}^{n}$ & 0 & $r$ & $r$ \\
$\mathbb{P}^{n}$ & +1 & $\sin r$ & $\tan r$ \\
\hline
\end{tabular}

Note that counterexamples of Helgason show that condition (1) is necessary for the result.

\section{LINK BETWEEN THE RADON TRANSFORMS}

In this section we prove Theorem 2.1, which makes a connection between the $k$-dimensional Radon transform on the Euclidean space and the $k$-dimensional totally geodesic Radon transform on the constant curvature space $\mathscr{M}^{n} \quad(1 \leq$ $k \leq n-1)$. Recently and independently this connection was discovered for the hyperbolic spaces in [1], where it is used to get range characterizations.

For convenience we use geodesic polar coordinatization for all the constant curvature spaces considered. For this reason, after fixing a point $O \in \mathscr{M}^{n}$ we write the function $f$ in the form $f(\omega, r)=f\left(\operatorname{Exp}_{o} r \omega\right)$, where $\omega \in T_{o} \mathscr{M}^{n}$ is a unit vector and $r$ is the distance coordinate. For a $k$-dimensional totally geodesic submanifold $\xi(1 \leq k \leq n-1)$ we shall use the notation $|\xi|$ to denote the distance of $\xi$ from $O$.

Theorem 2.1. If $f \in L^{2}\left(\mathbb{R}^{n}\right)$ and $g\left(\tilde{\mu}^{-1}(x)\right)=f(x)\left(1+\kappa|x|^{2}\right)^{(k+1) / 2}$, then

$$
\left(R_{k} f\right)(\xi)={\sqrt{1+\kappa|\xi|^{2}}}^{-1}\left(\widetilde{R}_{k} g\right)\left(\tilde{\mu}^{-1}(\xi)\right),
$$

where $1 \leq k \leq n-1, R_{k}$ (resp. $\widetilde{R}_{k}$ ) denotes the $k$-dimensional Radon transform on $\mathbb{R}^{n}$ (resp. $\left.\mathscr{M}^{n}\right) ; \xi$ is a k-dimensional hyperplane in $\mathbb{R}^{n}, x \in \mathbb{R}^{n}$; $\tilde{\mu}^{-1}$ is the inverse of $\tilde{\mu}$; and $\kappa \in\{-1,0,+1\}$ is the curvature of $\mathscr{M}^{n}$. In the hyperbolic case we require supp $f \subset B^{n}$, where $B^{n}$ is the unit ball in $\mathbb{R}^{n}$. 
Proof. Taking the $(k+1)$-dimensional totally geodesic submanifold in $\mathbb{R}^{n}$ (resp. $\mathscr{M}^{n}$ ) spanned by $O$ and $\xi$ (resp. $\tilde{\mu}^{-1}(\xi)$ ) and restricting the Radon transform to this spanned submanifold one can assume that $k+1=n$. We do this and omit the index $k$ of $R$ and $\widetilde{R}$.

According to [10, Theorem 4.1] for $g \in L^{2}\left(\mathscr{M}^{n}\right)$ we have

$$
\tilde{R} g(\bar{\omega}, h)=\int_{S_{\bar{\omega}, \cos \alpha_{0}(h)}^{n-1}} g\left(\omega, \mu^{-1}\left(\frac{\mu(h)}{\langle\omega, \bar{\omega}\rangle}\right)\right) \frac{\left(\langle\omega, \bar{\omega}\rangle / \mu^{2}(h)+\kappa\right)^{-n / 2}}{\nu(h)} d \omega,
$$

where we used the polar coordinates $(\bar{\omega}, h)$ of the point $\operatorname{Exp}_{o}(h \bar{\omega})$ of $\xi$ nearest to the origin $O$. Further on we shall call $(\bar{\omega}, h)$ the polar coordinates of $\xi$. $S^{n-1}$ is the unit sphere in $T_{O} \mathscr{M}^{n}$, and $d \omega$ is the surface measure on it. For $t \in[-1,+1)$

$$
S_{\bar{\omega}, t}^{n-1}=\left\{\omega \in S^{n-1}:\langle\omega, \bar{\omega}\rangle>t\right\},
$$

where $\langle\cdot, \cdot\rangle$ is the standard scalar product in $T_{O} \mathscr{M}^{n}$ identified with $\mathbb{R}^{n}$. Finally $\alpha_{0}(h)$ is the half of the angle that the submanifold $\xi$ subtends at the origin $O$. (Let $\gamma$ be the unique geodesic through $O$ perpendicular to $\xi$. For any point $P \in \xi$ let $\gamma(P)$ denote the geodesic joining $P$ and $O$. The angle of $\gamma$ and $\gamma(P)$ will tend to $\alpha_{0}(h)$ if $P$ goes to a "farthest" point of $\xi$. Thus $\alpha_{0}(h) \equiv \frac{\pi}{2}$ for $\kappa=+1$ or $\kappa=0$, and $\alpha_{0}(h)=\arccos \tanh h$ for $\kappa=-1$.) $\mu, \nu$ and $\kappa \in\{-1,0,+1\}$ are set according to $\mathscr{M}^{n}$, of course.

As is well known, or take equation (2.2) for the special entries $\kappa=0, \mu(r)=$ $r$, and $\nu(r)=r$, the Euclidean Radon transform $R$ is

$$
R f(\bar{\omega}, h)=\int_{S_{\bar{\omega}, 0}^{n-1}} f\left(\omega, \frac{h}{\langle\omega, \bar{\omega}\rangle}\right) \frac{h^{n-1}}{\langle\omega, \bar{\omega}\rangle^{n}} d \omega .
$$

Let the polar coordinates of $\xi$ be $(\bar{\omega}, h)$ in $\mathbb{R}^{n}$, and then observe that the polar coordinates of $\tilde{\mu}^{-1}(\xi)$ are $\left(\bar{\omega}, \mu^{-1}(h)\right)$. Substituting into equation (2.2) the function $g(\omega, r)=f(\omega, \mu(r))\left(1+\kappa \mu^{2}(r)\right)^{n / 2}$, which is $g\left(\tilde{\mu}^{-1}(x)\right)=$ $f(x)\left(1+\kappa|x|^{2}\right)^{(k+1) / 2}$ in polar coordinates, we obtain

$$
\tilde{R} g(\bar{\omega}, h)=\int_{S_{\bar{\omega}, \cos \alpha_{0}(h)}^{n-1}} f\left(\omega, \frac{h}{\langle\omega, \bar{\omega}\rangle}\right) \frac{\left(1+\kappa h^{2} /\langle\omega, \bar{\omega}\rangle\right)^{n / 2}}{\left(\langle\omega, \bar{\omega}\rangle / h^{2}+\kappa\right)^{n / 2}} \frac{1}{\nu\left(\mu^{-1}(h)\right)} d \omega .
$$

Since $\frac{\mu(r)}{\nu(r)}=\sqrt{1+\kappa \mu^{2}(r)}$, this equation immediately gives (take $r=\mu^{-1}(h)$ ) the formula of the theorem. (Note that $\alpha_{0}(h)$ can be less than $\frac{\pi}{2}$ only in the hyperbolic case, but then the support of $f$ is appropriately restricted to this angle.)

We note here that by Beltrami's theorem the only Riemannian manifolds that have geodesic correspondences between each other are the spaces of constant curvature; therefore, similar connections between Radon transforms on more general spaces are unlikely.

\section{THE SUPPORT THEOREMS}

In principle, in this section we only transpose the known results Lemma 1.1 and Theorem 1.2 from their spaces to other spaces via Theorem 2.1. 
Theorem 3.1. Let $k \leq n-2$, and assume $g \in C\left(\mathscr{M}^{n}\right)$ satisfies the following conditions:

(i) The function $g(\omega, r) \nu^{k+1}(r)$ tends to a finite number $G(\omega)$ for each $\omega \in$ $S^{n-1} \subset T_{O} \mathscr{M}^{n}$ as

$$
\begin{cases}r \rightarrow \frac{\pi}{2} & \text { if } \kappa=+1 \\ r \rightarrow \infty & \text { if } \kappa=0,-1\end{cases}
$$

and $G(\omega)=G(-\omega)$.

(ii) $\widetilde{R}_{k} g(\xi)=0$ for each $k$-dimensional totally geodesic $\xi$ with $|\xi|>1$. Then $g(\omega, h)=0$ for $h>1$.

Proof. If $\kappa=+1$, consider the function $h: S^{n} \rightarrow \mathbb{R}$ defined on the open upper half of $S^{n}$ by $g(\omega, r)$, on the open bottom half of $S^{n}$ by the reflection of the upper half with respect to the center of $S^{n}$, and on the equator by $G(\omega)$. Then $h$ is a symmetric continuous function on $S^{n}$ because of (i) and because $\nu\left(\frac{\pi}{2}\right)=1 ;$ hence, Lemma 1.1 gives the assertion.

If $\kappa=0$, consider the function $h: \mathbb{P}^{n} \rightarrow \mathbb{R}$ defined by

$$
h\left(\tilde{\mu}^{-1}(x)\right)=g(x)\left(1+|x|^{2}\right)^{(k+1) / 2},
$$

where $\tilde{\mu}$ corresponds to $\mathbb{P}^{n}$. By Theorem 2.1 , then, $\left(R_{k} g\right)(\xi) \sqrt{1+|\xi|^{2}}=$ $\left(\widetilde{R}_{k} h\right)\left(\tilde{\mu}^{-1}(\xi)\right)$ derives. Thus to get the statement of the theorem we only have to verify if the function $h$ satisfies our theorems' condition (i). Using its definition in polar coordinates, $h(\omega, r)=g(\omega, \tan r)\left(1+\tan ^{2} r\right)^{(k+1) / 2}$, this is done by

$$
\begin{aligned}
\lim _{r \rightarrow \pi / 2} h(\omega, r) \sin ^{k+1} r & =\lim _{r \rightarrow \pi / 2} g(\omega, \tan r)\left(1+\tan ^{2} r\right)^{(k+1) / 2} \\
& =\lim _{t \rightarrow \infty} g(\omega, t)\left(1+t^{2}\right)^{(k+1) / 2}=\lim _{t \rightarrow \infty} g(\omega, t) t^{k+1}=G(\omega),
\end{aligned}
$$

where the last equation comes from just the condition (i) on $g$.

If $\kappa=-1$, consider the function $h: B^{n} \rightarrow \mathbb{R}$ defined by $g\left(\tilde{\mu}^{-1}(x)\right)=$ $h(x)\left(1-|x|^{2}\right)^{(k+1) / 2}$. Then

$$
h(\omega, \tanh r)=\frac{g(\omega, r)}{\left(1-\tanh ^{2} r\right)^{(k+1) / 2}}
$$

in polar coordinates and Theorem 2.1 gives $\left(R_{k} h\right)(\xi) \sqrt{1-|\xi|^{2}}=\left(\widetilde{R}_{k} g\right)\left(\tilde{\mu}^{-1}(\xi)\right)$. Thus the statement of the theorem will follow after the verification of our theorem's condition (i) on $h$. To do this an easy observation is

$$
\begin{aligned}
\lim _{t \rightarrow 1} h(\omega, t) & =\lim _{r \rightarrow \infty} \frac{g(\omega, r)}{\left(1-\tanh ^{2} r\right)^{(k+1) / 2}} \\
& =\lim _{r \rightarrow \infty} g(\omega, r) \cosh ^{k+1} r=\lim _{r \rightarrow \infty} g(\omega, r) \sinh ^{k+1} r=G(\omega) .
\end{aligned}
$$

Thus $h$ can be extended to a function $h_{*}$ onto the closed unit ball so that it is uniformly continuous. Then $h_{*}$ extends continuously on to $\mathbb{R}^{n}$ by the Tietze extension theorem so that the extended function $h_{* *}$ has compact support. This function, $h_{* *}$, obviously satisfies condition (i) of our theorem, which completes the proof.

Now we turn to the Radon transforms integrating on the 1-codimensional total geodesic submanifolds. 
Theorem 3.2. Let $g \in C\left(\mathscr{M}^{n}\right)$ satisfy the following conditions $(k=n-1)$ :

(i-) If $\kappa=-1$, the function $g(\omega, r) \sinh ^{n}(r)$ tends to a finite number for each $\omega \in S^{n-1} \subset T_{O} \mathbf{H}^{n}$ as $r \rightarrow \infty$.

$\left(\mathrm{i}^{+}\right)$If $\kappa=0,+1$, the function $g(\omega, r) \mu^{m}(r)$ is bounded for each integer $m>0$.

(ii) $\widetilde{R} g(\xi)=0$ for each $(n-1)$-dimensional totally geodesic $\xi$ if $|\xi|>1$.

Then $g(\omega, h)=0$ for $h>1$.

Proof. Let $f: \mathbb{R}^{n} \rightarrow \mathbb{R}$ be given by $g\left(\tilde{\mu}^{-1}(x)\right)=f(x)\left(1+\kappa|x|^{2}\right)^{n / 2}$ or in polar coordinates $g(\omega, r)=f(\omega, \mu(r))\left(1+\kappa \mu^{2}(r)\right)^{n / 2}$. Then by the continuity of $\mu^{-1}$ and $g$ the function $f$ is continuous. Note that in the hyperbolic case this continuity is meant only in the open unit ball $B^{n} \subset \mathbb{R}^{n}$.

If $\kappa=-1$, the proof goes in the same way as in the previous theorem, so we leave it to the reader.

If $\kappa=0,1$, condition $\left(\mathrm{i}^{+}\right)$directly guarantees the first condition of Theorem 1.2. To see this one only has to observe $\kappa=1$. Then $f(\omega, \tan r) \tan ^{m} r=$ $g(\omega, r) \cos ^{n} r \tan ^{m}(r)$, and this with condition $\left(\mathrm{i}^{+}\right)$gives the boundedness of $f(\omega, t) t^{m}$ for each integer $m>0$.

By Theorem 2.1

$$
(R f)(\xi) \sqrt{1+\kappa|\xi|^{2}}=(\widetilde{R} g)\left(\tilde{\mu}^{-1}(\xi)\right)
$$

hence, the function $f$ also satisfies the second condition of Theorem 1.2 for $\mu(1)$. Namely, if $|\xi|>\mu(1)$, then $\left|\tilde{\mu}^{-1}(\xi)\right|>1$ since the function $\mu^{-1}$ is strictly increasing. Therefore, for such an $(n-1)$-dimensional hyperplane $\xi$, $|\xi|>\mu(1)$, we have $\widetilde{R} g\left(\tilde{\mu}^{-1}(\xi)\right)=0$ by condition (ii), so $R f(\xi)=0$, too.

Now Theorem 1.2 implies that $f(x)$ must be zero for $|x|>\mu^{-1}(1)$; hence, $g(\omega, r)=0$ follows for $|r|>1$. This proves the theorem.

Note that Theorem 3.1 is just Lemma 1.1 in the case of the sphere $(\kappa=+1)$ and Theorem 3.2 is equivalent to Theorem 1.2 in the case of the Euclidean space $(\kappa=0)$.

For the hyperbolic space Theorem 2.2 gives a considerable improved version of Helgason's similar theorem [4-7] requiring only a decrease of order $n$ if $k=n-1$. I do not know any other result for $k \leq n-2$. It is interesting to note that there is no difference between the cases $k=n-1$ and $k \leq n-2$ !

For the sphere our condition in the case $k=n-1$ is equivalent to the function being $C^{\infty}$ at the equator, formed by the points having $\frac{\pi}{2}$ distance from the point $O$, and all of its derivatives being zero there. This condition cannot be omitted, because the decay condition in Theorem 1.2 is known to be necessary. Theorem 3.2 therefore shows that the condition imposed in $[6,7]-$ that $g$ should vanish on the equator along with its odd order derivatives (since $g$ is symmetric)-is not sufficient for the conclusion. As is known, support theorems on the sphere had been proved before only with the assumption that the function vanishes in some belt around the equator $[9,11]$.

In the most classical case, on the Euclidean space, Theorem 3.1 gives the support theorem for $k \leq n-2$, requiring the function to decrease only of order $k+1$, which seems to have connection with Solmon's [13, Theorem 7.7]. This relation will be detailed in a forthcoming paper of Berenstein, Casadio Tarabusi, and Kurusa. 


\section{ACKNOWLEDGMENT}

The author thanks Z. I. Szabó for pointing out the differences between the cases $k=n-1$ and $k \leq n-2$.

\section{REFERENCES}

1. C. A. Berenstein and E. C. Tarabusi, Range of the $k$-dimensional Radon transform in real hyperbolic spaces, preprint.

2. J. Boman and E. T. Quinto, Support theorems for real analytic Radon transforms, Duke Math. J. 55 (1987), 943-948.

3. I. M. Gelfand, M. I. Graev, and N. Ya. Vilenkin, Generalized functions, Vol. 5, Academic Press, New York, 1966.

4. S. Helgason, Support of Radon transforms, Adv. Math. 38 (1980), 91-100.

5. —, The Radon transform, Birkhäuser, Boston, 1980.

6. __ The totally geodesic Radon transform on constant curvature spaces, Contemp. Math., vol. 113, Amer. Math. Soc., Providence, RI, 1990, pp. 141-149.

7. __ Support theorems in integral geometry and their applications, preprint.

8. A. Kurusa, The Radon transform on hyperbolic space, Geom. Dedicata 40 (1991), 325-339.

9. __ The Radon transform on half sphere, Acta Math. Sci. Szeged 58 (1993), 143-158.

10. Math. Scand. 70 (1992), 112-126.

11. E. T. Quinto, The invertibility of rotation invariant Radon transforms, J. Math. Anal. Appl. 91 (1983), 510-522.

12. R. Schneider, Functions on a sphere with vanishing integrals over certain subspheres, J. Math. Anal. Appl. 26 (1969), 381-384.

13. D. C. Solmon, Asymptotic formulas for the dual Radon transform and applications, Math. Z. 195 (1987), 321-343.

14. Wu-Yi Hsiang, On the laws of trigonometry of two-point homogeneous spaces, Ann. Global Anal. Geom. 7 (1989), 29-45.

Bolyai Institute, ARAdi vertanúk tere 1., H-6720 SZEged, Hungary

E-mail address: kurusa@inf .jate.u-szeged.hu 